University of South Carolina

Scholar Commons

$5-23-2005$

\title{
Spin Photovoltaic Effect in Quantum Wires with Rashba Interaction
}

Yuriy V. Pershin Dr

University of South Carolina - Columbia, pershin@physics.sc.edu

Carlo Piermarocchi

Follow this and additional works at: https://scholarcommons.sc.edu/phys_facpub

Part of the Physics Commons

\section{Publication Info}

Published in Applied Physics Letters, ed. Nghi Q. Lam, Volume 86, Issue 21, 2005, pages 212107-1-212107-3.

Pershin, Y. V., \& Piermarocchi, C. (2005). Spin photovoltaic effect in quantum wires with Rashba interaction. Applied Physics Letters, 86(21), 212107-1 - 212107-3. DOI: 10.1063/1.1935747

(C) Applied Physics Letters, 2005, American Institute of Physics http://apl.aip.org/resource/1/applab/v86/i21/p212107_s1

This Article is brought to you by the Physics and Astronomy, Department of at Scholar Commons. It has been accepted for inclusion in Faculty Publications by an authorized administrator of Scholar Commons. For more information, please contact digres@mailbox.sc.edu. 


\title{
Spin photovoltaic effect in quantum wires with Rashba interaction
}

\author{
Yuriy V. Pershin ${ }^{\text {a) }}$ and Carlo Piermarocchi \\ Department of Physics and Astronomy, Michigan State University, East Lansing, Michigan 48824-2320
}

(Received 4 February 2005; accepted 13 April 2005; published online 19 May 2005)

\begin{abstract}
We propose a mechanism for spin-polarized photocurrent generation in quantum wires. The effect is due to the combined effect of Rashba spin-orbit interaction, external magnetic field, and microwave radiation. The time-independent interactions in the wire give rise to a spectrum asymmetry in $k$ space. The microwave radiation induces transitions between spin-splitted subbands, and, due to the peculiar energy dispersion relation, charge and spin currents are generated at zero-bias voltage. We demonstrate that the generation of pure spin currents is possible under an appropriate choice of external control parameters. (C) 2005 American Institute of Physics.
\end{abstract}

[DOI: $10.1063 / 1.1935747]$

The Rashba spin-orbit interaction (SOI) (Ref. 1) in transport and equilibrium phenomena ${ }^{2-8}$ plays a central role in the fast growing fields of spintronics and quantum computation. ${ }^{9}$ In particular, it was recently discovered that the joint action of the Rashba SOI and in-plane magnetic field on electrons confined in one-dimensional quantum wires $(\mathrm{QW})$ results in unique properties. $^{2-4}$ Several useful applications based on these properties were proposed, including a scheme for measuring nuclear spin polarization ${ }^{2}$ and a spin filter. ${ }^{3}$

In this letter, we theoretically investigate the effect of a microwave radiation in a QW with Rashba SOI and an inplane magnetic field. This setup was stimulated by recent experiments on the modifications of the Hall effect in the presence of a microwave field. ${ }^{10}$ We will show below that spin and charge photocurrents can be generated in microwave irradiated QWs (spin photovoltaic effect). The effect originates in the broken symmetry of QW subbands caused by the interplay of SOI and constant magnetic field. We emphasize that this mechanism is based primarily on spin degrees of freedom in contrast to other mechanisms of the photovoltaic effect considered before (see, e.g., Ref. 11) and differs from the optical spin current generation. ${ }^{12}$

In our model, a ballistic QW of length $L$ is connected to two electron reservoirs having equal chemical potentials $\mu$. This geometry is realized, for instance, in a QW created by a split gate technique in a two-dimensional electron gas. For the sake of simplicity, we assume that only the QW region is irradiated by the microwave field. Without microwave radiation, the currents in QW from the left to the right reservoir and from the right to the left reservoir balance each other so that the total current through the $\mathrm{QW}$ is zero. The microwave radiation induces transitions between spin-splitted subbands. The electron wave vector $k$ is conserved in such transitions, however, in the presence of SOI, the electron velocity is not simply proportional to $k$. The direction of the electron velocity, in specific intervals of $k$, can be reversed after the transition. The intersubband transition rate, due to the asymmetry of QW subbands, is different for left- right-moving electrons, and produces a net charge current. The spin current is also influenced by the microwave field, since spin flips occur in these transitions.

\footnotetext{
${ }^{a)}$ Electronic mail: pershin@pa.msu.edu
}

We consider a QW in the $x$ direction created via a lateral confinement (in the $y$ direction) of a 2DEG in the $(x, y)$ plane. The Hamiltonian for the conduction electrons in the QW in the presence of the microwave radiation can be written in the form, ${ }^{2-4}$

$$
H=\frac{\mathbf{p}^{2}}{2 m^{*}}+V(y)-i \alpha \sigma_{y} \frac{\partial}{\partial x}+\frac{g^{*} \mu_{B}}{2} \boldsymbol{\sigma} \cdot \mathbf{B}(t)+U(z, t) .
$$

We assume the microwave field propagating in the $(x, y)$ plane and we fix the electric-field component in the $z$ direction. In Eq. (1), $\mathbf{p}$ is the momentum of the electron, $m^{*}$ is the electron effective mass, $V(y)$ is the lateral confinement potential due to the gates, $\alpha$ is the SOI constant, $\boldsymbol{\sigma}$ is the vector of Pauli matrices, $\mu_{B}$ and $g^{*}$ are the Bohr magneton and effective $g$ factor, and $U(z, t)$ is the potential due to the electric field component of the radiation. $\mathbf{B}=\mathbf{B}_{0}+\mathbf{B}_{1} \cos (\omega t)$, where $\mathbf{B}_{0}$ is the in-plane constant magnetic field and $\mathbf{B}_{1}$ is the magnetic-field component of the microwave radiation, and $\omega$ is the radiation frequency. $U(z, t)$ can be neglected because it does not couple spin-splitted subbands. The third term in Eq. (1) represents the Rashba SOI for an electron moving in the $x$ direction. We assume that the effects of the Dresselhaus SOI can be neglected. ${ }^{13}$

At $B_{1}=0$, the solutions of the Schrödinger equation can be written in the form

$$
\Psi_{l, \pm}(k)=\frac{e^{i k x}}{\sqrt{2}}\left(\begin{array}{c} 
\pm e^{i \varphi} \\
1
\end{array}\right) \phi_{l}(y)
$$

where $\varphi=\arctan \left[-B_{0, y} / B_{0, x}-2 \alpha k /\left(g^{*} \mu_{B} B_{0, x}\right)\right]$ and $\phi_{l}(y)$ is the wave function of the transverse modes [due to the confinement potential $V(y)]$. The eigenvalue problem can be solved to obtain

$$
E_{\ell, \pm}(k)=\frac{E_{Z}^{2}}{E_{\alpha}} \widetilde{k}^{2}+E_{l}^{\mathrm{tr}} \pm E_{Z} \sqrt{1+\frac{2 \widetilde{k} B_{0, y}}{B_{0}}+\widetilde{k}^{2}} .
$$

Here, $\tilde{k}=k \alpha / E_{Z}, E_{\alpha}=2 m^{*} \alpha^{2} / \hbar^{2}, E_{Z}=g^{*} \mu_{B} B_{0} / 2$, and $E_{l}^{\mathrm{tr}}$ is the $l$ th eigenvalue of $V(y)$. Assuming the parabolic confinement potential in the $y$ direction, we have $E_{l}^{\mathrm{tr}}=\hbar \omega_{0}(l+1 / 2)$.

The energy spectrum corresponding to Eq. (3) is illustrated in Fig. 1, for the two spin-split subbands characterized by $l=0$ and $\mathbf{B}_{0}$ directed at $\pi / 4$ with respect to the $x$ axis in the $(x, y)$ plane. Notice that the down-splitted subband has a 


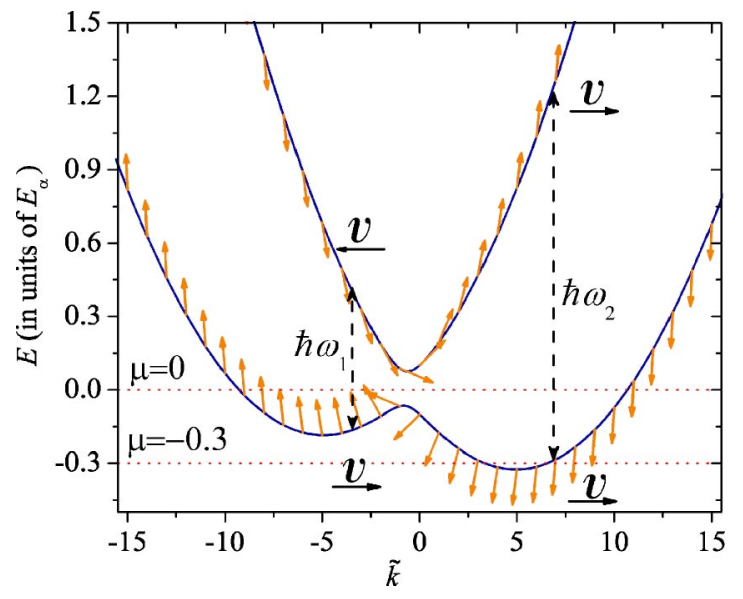

FIG. 1. (Color online) Energy dispersion $E_{0, \pm}(k)$ (with respect to $E_{0}^{\mathrm{tr}}$ ) for $E_{Z}=0.1 E_{\alpha}$ and $\mathbf{B}_{0}=\left(B_{0} / \sqrt{2}, B_{0} / \sqrt{2}, 0\right)$. Spin orientation is illustrated by arrows $\left[\left\langle\sigma_{x}\right\rangle\right.$ is plotted along $\tilde{k},\left\langle\sigma_{y}\right\rangle$ is plotted along $E$, and $\left.\left\langle\sigma_{z}\right\rangle=0\right]$.

clearly defined asymmetry. This subband features several local extrema, namely, two minima and one maximum. The energy branches avoid the crossing and form a local gap. The expectation values of spin polarization in the states (2) are $\left\langle \pm\left|\sigma_{x}\right| \pm\right\rangle= \pm \cos [\varphi(k)]$ and $\left\langle \pm\left|\sigma_{y}\right| \pm\right\rangle=\mp \sin [\varphi(k)]$. While the external magnetic field realigns the electron spins in the gap region, far from this region the spins are polarized in $y$ direction by the Rashba SOI (Fig. 1). The velocity of an electron is determined by $v_{ \pm}(k)=\partial E_{ \pm} / \hbar \partial k$. Denoting by $k_{+}^{\mathrm{min}}$, $k_{-}^{\min , 1}, k_{-}^{\max }, k_{-}^{\min , 2}$ the positions of the local extrema of $E_{l,+}$ and $E_{l,-}$, we find from Eq. (3) that $v_{+}<0$ for $k<k_{+}^{\mathrm{min}}, v_{+}$ $>0$ for $k>k_{+}^{\min }, v_{-}<0$ for $k<k_{-}^{\min , 1}$ and $k_{-}^{\max }<k<k_{-}^{\min , 2}$, $v_{-}>0$ for $k_{-}^{\text {min, } 1}<k<k_{-}^{\max }$ and $k>k_{-}^{\min , 2}$. Generally, $k_{+}^{\text {min }}$ $\neq k_{-}^{\max }$. Thus, there are two intervals of $k$ when the directions of $v_{-}(k)$ and $v_{+}(k)$ are opposite.

Next, we consider transitions generated by the timedependent magnetic field $\mathbf{B}_{1} \cos (\omega t)$. It can be shown that the transition rate differs from zero only for transitions between subbands characterized by the same number $l$ with conservation of $k$ (examples of such vertical transitions are presented in Fig. 1). We calculate the transition rate using the expression

$$
W=\frac{2 \pi}{\hbar}\left|\left\langle+\left|\frac{g^{*} \mu_{B}}{2} \boldsymbol{\sigma} \mathbf{B}_{1}\right|-\right\rangle\right|^{2} \delta\left(E_{l,+}-E_{l,-}-\hbar \omega\right) .
$$

It follows from Eq. (4) that the transition rate depends on the relative orientation of the spin polarization in a state $k$ and the direction of $\mathbf{B}_{1}$. This property allows one to use the direction of the oscillating magnetic field as an additional parameter that controls spin and charge currents in the QW.

In order to take into account the effect of the timedependent magnetic field on transport properties of the QW we solve the Boltzmann equations

$$
v_{\mp}(k) \frac{\partial f_{\mp}}{\partial x}=W f_{ \pm}\left(1-f_{\mp}\right)-W f_{\mp}\left(1-f_{ \pm}\right),
$$

for the distribution functions $f_{ \pm}(k, x)$. Assuming that the chemical potential is below the minimum of $E_{+}(k)$ and a low temperature, we supplement Eq. (5) by the following boundary conditions: For $v_{-}(k)>0, f_{-}(x=0)=f(\mu)$; for $v_{-}(k)<0$, $f_{-}(x=L)=f(\mu)$; for $v_{+}(k)>0, f_{+}(x=0)=0 ;$ for $v_{+}(k)<0$, $f_{+}(x=L)=0$, where $f(\mu)$ is the Fermi function. The solution

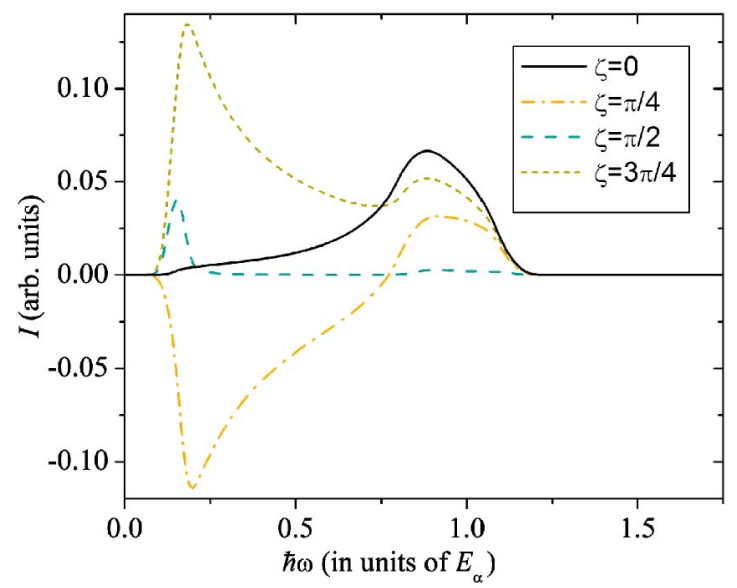

FIG. 2. (Color online) Current through QW as a function of the excitation frequency $\omega$. $\zeta$ is the in-plane angle between $\mathbf{B}_{1}$ and $x$ axis, $\mu=0, T=0$.

of linearized Eq. (5) with the specified above boundary conditions was found analytically. We calculate the current as: ${ }^{14}$ $I=\frac{e}{h} \sum_{\nu= \pm} \int_{-\infty}^{\infty} v_{\nu}(k) f_{\nu}(k) d k$. We found that the transitions conserving the velocity direction do not change the charge current. The current as a function of the excitation frequency is shown in Fig. 2 and has a two-peak structure. The first peak in this structure corresponds to transitions between the states with $k$ near $k_{-}^{\max }$, the second peak corresponds to transitions between states with $k$ near $k_{-}^{\mathrm{min}, 2}$. The direction and amplitude of the charge photocurrent shows a significant dependence on the direction of $\mathbf{B}_{1}$, especially in the first peak region, because the spin structure in this region is strongly affected by the magnetic field direction.

As electrons carry spin as well as charge, the timedependent magnetic field also influences the spin current through the wire. The spin current can be defined as the transport of electron spins in real space. When the electron transport is confined to one dimension, the spin current is a vector. Its components can be calculated using $I_{\gamma}^{s}$ $=\sum_{\nu= \pm} \int_{-\infty}^{\infty}\left\langle\nu\left|\sigma_{\gamma}\right| \nu\right\rangle v_{\nu}(k) f_{\nu}(k) d k$ where $\gamma=(x, y, z)$. Figure 3 shows the $x$ and $y$ components of the spin current $\left(I_{z}^{s}=0\right)$ calculated for two different values of the chemical potential $\mu$. The main features of the spin current are: (i) The spin current is coordinate dependent; (ii) transitions conserving the direction of the electron velocity also contribute to the spin current; (iii) generation of a pure spin current (without a charge current) occurs for transitions with $v_{-}(k) v_{+}(k)>0$. The spin current components calculated at $\mu=0$ have a complex dependence on $\omega$. At $\mu=-0.3 E_{\alpha}$, the role of different transitions can be more easily understood. With increase of $\omega$, we first observe excitations with $v_{-}(k) v_{+}(k)<0$, and, then, after passing the second minimum of $E_{-}(k)$ (see Fig. 1), with $v_{-}(k) v_{+}(k)>0$. The insets in Fig. 3 show that the first type of transitions leads to changes in spin current at $x=0$ and $x=L$, while the second type of transitions changes the spin current at $x=L$ only. The asymmetry of the spin current components at $x=0$ and $x=L$ is a signature of pure spin currents in the system.

We now discuss the conditions for an experimental observation of this spin photovoltaic effect. First, a QW should be fabricated from a structure with large Rashba SOI. A promising candidate are InAs-based semiconductor heterostructures, which have a relatively large $\alpha{ }^{15}$ The characteristic energy of the SOI for these structures $(\alpha=4.5$ 

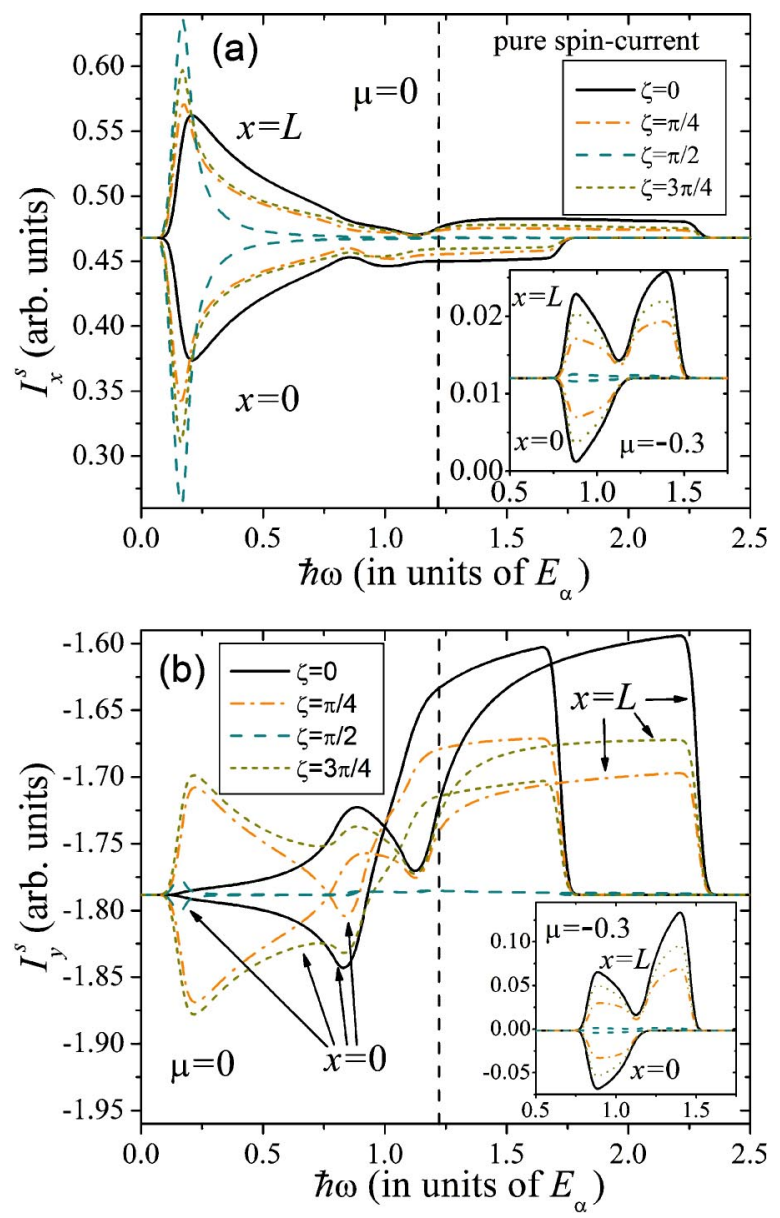

FIG. 3. (Color online) Spin current components as a function of the excitation frequency $\omega, \mu=0, T=0$. The region of pure spin currents is to the right of the vertical dashed line. Insets: Spin current components at $\mu=-0.3$.

$\left.\times 10^{-11} \mathrm{eV} \mathrm{m}, m^{*}=0.036 m_{e}\right)^{16}$ is $E_{\alpha}=1.9 \mathrm{meV}$. Assuming $E_{Z}=0.1 E_{\alpha}$ and taking $g^{*}=6,{ }^{17}$ we obtain $B_{0}=1.1 \mathrm{~T}$. Second, we note that extremely low temperatures are not required for experimental observation of the spin photovoltaic effect. From the condition $k_{B} T \lesssim E_{\alpha}, E_{Z}$, we estimate $T \lesssim 2 \mathrm{~K}$. Finally, the condition that $B_{1} \neq 0$ only in the $\mathrm{QW}$ region was used only for convenience. In a real experiment, the whole system (QW and leads) is subjected to a finite $B_{1}$. The effect of the leads depends on the particular system studied, but should not considerably affect the scheme proposed here, especially if there is no appreciable SOI in the leads. The spin and charge currents can be measured by any appropriate experimental technique. We believe that the charge current can be measured in a standard way, for example, using a sensitive amperemeter. The most convenient technique for spin current measurement is the scanning Kerr rotation spectroscopy, which was recently employed in the detection of the spin Hall effect in semiconductors. ${ }^{18}$

This research was supported by the National Science Foundation, Grant NSF DMR-0312491.

${ }^{1}$ Y. A. Bychkov and E. I. Rashba, JETP Lett. 39, 78 (1984).

${ }^{2}$ J. A. Nesteroff, Y. V. Pershin, and V. Privman, Phys. Rev. Lett. 93, 126601 (2004).

${ }^{3}$ P. Středa and P. Šeba, Phys. Rev. Lett. 90, 256601 (2003).

${ }^{4}$ J. A. Nesteroff, Y. V. Pershin, and V. Privman, Phys. Rev. B 69, 121306(R) (2004).

${ }^{5}$ M. Valn-Rodrguez, A. Puente, and L1. Serra, Phys. Rev. B 69, 153308 (2004).

${ }^{6}$ J. Splettstoesser, M. Governale, and U. Zülicke, Phys. Rev. B 68, 165341 (2003).

${ }^{7}$ S. Saikin, M. Shen, and M.-C. Cheng, IEEE Trans. Nanotechnol. 3, 173 (2004).

${ }^{8}$ S. Debald and B. Kramer, cond-mat/0411444.

${ }^{9}$ Semicondutor Spintronics and Quantum Computation, edited by D. D. Awschalom, D. Loss, and N. Samarath (Springer, Berlin, 2002).

${ }^{10}$ R. G. Mani, J. H. Smet, K. von Klitzing, V. Narayanamurti, W. B. Johnson, and V. Umansky, Nature (London) 420, 646 (2002).

${ }^{11}$ L. Fedichkin, V. Ryzhii, and V. Vyurkov, J. Phys.: Condens. Matter 5, 6091 (1993).

${ }^{12}$ R. D. R. Bhat and J. E. Sipe, Phys. Rev. Lett. 85, 5432 (2000).

${ }^{13}$ Y. Ohno, R. Terauchi, T. Adachi, F. Matsukura, and H. Ohno, Phys. Rev. Lett. 83, 4196 (1999).

${ }^{14}$ S. Datta, Electronic Transport in Mesoscopic Systems (Cambridge University Press, Cambridge, UK, 1995).

${ }^{15}$ R. H. Silsbee, J. Phys.: Condens. Matter 16, R179 (2004).

${ }^{16}$ D. Grundler, Phys. Rev. Lett. 84, 6074 (2000).

${ }^{17}$ C. H. Möller, C. Heyn, and D. Grundler, Appl. Phys. Lett. 83, 2181 (2003).

${ }^{18}$ Y. K. Kato, R. C. Mayer, A. C. Gossard, and D. D. Awschalom, Science 306, 1910 (2004). 